\title{
ESTRUTURA DE ESPÉCIES ARBÓREAS SOB EFEITO DE BORDA EM UM FRAGMENTO DE FLORESTA ESTACIONAL SEMIDECIDUAL EM PERNAMBUCO ${ }^{1}$
}

\begin{abstract}
Alan Caue de Holanda², Ana Lícia Patriota Feliciano ${ }^{3}$, Luiz Carlos Marangon ${ }^{3}$, Moisés Silva dos Santos $^{4}$, Cybelle Laís Souto Maior Sales de Melo ${ }^{4}$ e Mayara Maria de Lima Pessoa ${ }^{4}$

RESUMO - Os fragmentos florestais são considerados os únicos redutos detentores de biodiversidade do planeta. Conhecer os processos que decorrem após a fragmentação, a exemplo da estrutura arbórea ocorrente na borda, é de fundamental importância para se proporem medidas conservacionistas. Os objetivos deste trabalho, desenvolvido em uma área de 83,8 ha, localizada no Município de Nazaré da Mata, PE, foram efetuar o levantamento fitossociológico de espécies arbóreas adultas sob efeito de borda e verificar a similaridade florística entre as parcelas. A área amostral foi de $10.000 \mathrm{~m}^{2}$, equivalentes à implantação de 10 transectos de 10 x 100 m perpendiculares à borda, distribuídos de forma sistemática. Foram amostrados, etiquetados e identificados todos os indivíduos arbóreos com CAP e" $15 \mathrm{~cm}$. Posteriormente, realizaram-se os cálculos dos parâmetros fitossociológicos e da similaridade florística. Neste estudo, amostraram-se 1.238 indivíduos, pertencentes a 72 táxons, distribuídos em 26 famílias botânicas. As espécies Campomanesia xanthocarpa, Zanthoxylum rhoifolium e Anadenanthera colubrina apresentaram o maior valor de importância, pois, teoricamente, conseguiram explorar melhor o recurso proporcionado pelo hábitat.
\end{abstract}

Palavras-chave: Fragmentação, Fitossociologia e Borda.

\section{EDGE EFFECT ON THE STRUCTURE OF TREE SPECIES IN A SEASONAL FOREST FRAGMENT IN PERNAMBUCO}

\begin{abstract}
Forest fragments are considered the last refuges for biodiversity in the planet. Knowing the processes that occur after fragmentation, such as the tree structure that occurs in the edge, is fundamental to propose conservationist measures. The objective of this work was to carry out a phytosociological survey of adult tree species under edge effect and verify the floristic similarity among the plots. The work was developed in an area of 83,8 ha located in the municipality of Nazaré da Mata-PE. The sample area comprised 10.000 $\mathrm{m}^{2}$, equivalent to the implantation of 10 contiguous transects of $10 \times 100 \mathrm{~m}$ perpendicular to the edge. All tree individuals with CAP e" $15 \mathrm{~cm}$ were sampled, labeled and identified. Afterward, phytosociological parameters and the floristic similarity were calculated. In this study, 1238 individuals, belonging to 72 táxons and distributed into 26 botanical families were sampled. The species Campomanesia xanthocarpa, Zanthoxylum rhoifolium, and Anadenanthera colubrina, showed the largest importance value, as theoretically, they managed to better exploit habitat resources.
\end{abstract}

Keywords: Fragmentation, Phytosociology and Edge.

\section{INTRODUÇÃO}

O Brasil é o país com maior área de florestas tropicais úmidas do mundo, cujos estudos revelam que os fatores de maior influência nas fitofisionomias das florestas brasileiras é o clima, variando de sempre-úmido ao de estação seca definida; a disponibilidade d'água no solo, distinguindo-se as florestas alagadas e as de terras secas (TANIZAKI e MOUL TON, 2000).

Para Veloso et al. (1992), o conceito ecológico de Florestas Estacionais Semideciduais relaciona-se com as condições climáticas da região de ocorrência,

\footnotetext{
${ }^{1}$ Recebido em 26.02.2008 e aceito para publicação em 14.10.2009.

${ }^{2}$ Programa de Pós-Graduação em Ciências Florestais na UFRPE . E-mail: <holandaac@yahoo.com.br>

${ }^{3}$ Departamento de Ciência Florestal da UFRPE. E-mail: <licia@dcfl.ufrpe.br> e <marangon@dcfl.ufrpe.br> 4 ????????
} 
caracterizada por apresentar duas estações distintas, uma chuvosa e outra seca, ou com acentuada variação térmica.

Diante de toda a biodiversidade nas mais diversas áreas fitofisionômicas do Brasil, há um processo que compromete parte dessa riqueza de espécies, que é a fragmentação florestal.

O processo de redução e isolamento da vegetação natural, conhecido por fragmentação florestal, tem consequências sobre a estrutura e processos das comunidades vegetais, além da evidente redução na área original dos hábitats. Estudos relataram extinções locais e alterações na composição e abundância de espécies que levam à alteração ou, mesmo, à perda de processos naturais das comunidades (MMA, 2003).

Conhecer os processos que decorrem da fragmentação de hábitats, como a criação de bordas, é fundamental para a elaboração de estratégias de recuperação de fragmentos florestais, as quais forneceriam subsídios para o desenho de reservas (RESTREPO et al., 1999) e medidas preventivas que evitem a extinção de espécies (AGUILAR e GALETTO, 2004).

A ruptura de um contínuo florestal, para a expansão de fronteiras agrícolas, é uma das principais causas da fragmentação. Em decorrência, há formação de bordas, expondo espécies adaptadas a determinados fatores abióticos, anteriormente do interior da floresta, a fatores adversos, como: altos índices de temperatura, luminosidade, velocidade dos ventos e baixa umidade relativa do ar. Por conseguinte, irá haver aumento da evapotranspiração, que influenciará o tombamento de indivíduos de porte maior.

Uma borda recém-criada, segundo Rodrigues e Nascimento (2006), em termos teóricos, pode ser estruturalmente homogênea ou muito semelhante ao interior florestal. Ao longo do tempo, irão ocorrer outras transformações, relacionadas em grande parte aos efeitos de borda, que podem resultar em uma comunidade mais heterogênea nesse limite.

A diversidade de espécies nas bordas pode variar fortemente entre os fragmentos devido à sua estrutura e ao seu isolamento, sendo negativa a relação entre diversidade de espécies e grau de isolamento (METZGER, 2000).

R. Árvore, Viçosa-MG, v.34, n.1, p.103-114, 2010
Outro fator agravante é o fato de que grande parte dos remanescentes de floresta se encontra em propriedades privadas, sendo tais ambientes geralmente muito vulneráveis a contínuos distúrbios decorrentes, principalmente, de uso não sustentável (SILVA et al., 2004).

Partindo desse princípio, o objetivo do trabalho foi avaliar a estrutura e similaridade florística de espécies arbóreas sob efeito de borda em um fragmento de Floresta Estacional Semidecidual, denominado Mata do Alcaparra, localizado no Município de Nazaré da Mata, em Pernambuco.

\section{MATERIAL E MÉTODOS}

\section{1. Área de estudo}

A área de estudo é um fragmento de Floresta Estacional Semidecidual denominado Mata do Alcaparra, pertencente à Usina Petribu S/A e localizada no Município de Nazaré da Mata, Pernambuco, com uma área de 83,8 ha, sob as coordenadas 7044'00" latitude Sul e 35 12 '30", longitude oeste (Figura 1). O solo é caracterizado como Podzólico Vermelho-Amarelo (EMBRAPA, 1999). A altitude média da área é em torno de $89 \mathrm{~m}$. O clima da região, segundo a classificação de Köppen, é o As', com precipitação média anual medidas nos últimos 20 anos de $1.578 \mathrm{~mm}$, com temperatura média anual de $29^{\circ} \mathrm{C}$ (AGENDA 21 NAZARÉ DA MATA, 2004).

A área onde se encontrava o fragmento estava inserida em uma matriz de cana-de-açúcar, possuindo aceiros (5 $\mathrm{m}$ de largura) apenas nos lados nordeste e sudeste, que serviram de estrada para escoamento desse produto; já no lado oeste não existia aceiro.

\subsection{Coleta de dados}

Para amostragem do componente arbóreo foram implantados 10 transectos de 10 X $100 \mathrm{~m}$, dispostos perpendicularmentes à borda, estando eles distribuídos em lados opostos e de forma sistemática, eqüidistantes a $153 \mathrm{~m}$, em linha reta, tomando-se como referência as extremidades no sentido norte-sul. Os transectos foram subdivididos em parcelas contíguas de $10 \times 10$ $\mathrm{m}$, para amostragem dos indivíduos arbóreos, totalizando 100 parcelas, correspondentes a uma área amostral de $10.000 \mathrm{~m}^{2}$.

Em cada parcela, foram amostrados e etiquetados com placas de alumínio todos os indivíduos arbóreos adultos com circunferência a altura do peito (CAP) 
e" $15 \mathrm{~cm}$, sendo estes mensurados com fita métrica, e a altura estimada com módulos de tesoura de alta poda, cujo comprimento de cada módulo era de $2 \mathrm{~m}$.

\subsection{Análise dos dados}

Para caracterizar a estrutura horizontal da borda do fragmento florestal, foram analisados os parâmetros fitossociológicos: Densidades, frequências, dominâncias, valor de cobertura e valor de importância (MARTINS, 1993), com o auxílio do Software Mata Nativa versão 2.0, no intuito de obter maior precisão e confiabilidade nos dados do componente arbóreo.

Na análise da distribuição diamétrica dos 10 transectos, fez-se um gráfico com o número de árvores por classe de diâmetro, com amplitudes de classe de $2,5 \mathrm{~cm}$, em todos os indivíduos adultos, amostrados na área. O diâmetro mínimo considerado foi de 4,7 cm.

Para a estrutura vertical foi gerada uma figura com número de indivíduos e área basal por classes de altura nos eixos das ordenadas. No eixo das abscissas, foram consideradas as alturas: $\mathrm{H}<4,32 \mathrm{~m} ; 4,32 \mathrm{~m} \mathrm{~d}$ " $\mathrm{H}<9,16 \mathrm{~m}$; e H e" 9,16 m.

No cálculo da diversidade florística foi utilizado o índice de diversidade de Shannon-Weaner $\left(\mathrm{H}^{\circ}\right)$ (MUELLER-DOMBOIS e ELLEMBERG, 1974).

A similaridade florística foi feita a partir da formação de uma matriz de presença e ausência de espécies, utilizando a distância euclidiana como medida de dissimilaridade e o algoritmo de agrupamento de Ward. As listas de espécies dos quatro fragmentos foram convertidas em uma matriz de presença/ausência, e fez-se uma ordenação por meio de uma análise de correspondência retificada Detrendend Correspondence Analysis - DCA (CAUSTON, 1988). Para analisar os dados, foi utilizado o programa Pc-Ord for Windows versão 4.14 (MCCUNE e MEFFORD, 1999).

\section{RESULTADOS E DISCUSSÃO}

Neste estudo foram amostrados 1.238 indivíduos, no componente arbóreo, pertencentes a 72 táxons, sendo 9 identificados em nível de família, 7 em nível de gênero, 52 em nível de espécie e 4 indeterminados.
Os indivíduos amostrados estão distribuídos em 26 famílias botânicas, sendo as famílias com maiores representatividades de indivíduos em porcentagem: Myrtaceae, com 23,95\%, seguida de Fabaceae com $13,81 \%$; Rutaceae com $13,32 \%$, Sapindaceae com 8,72\%, Erythroxylaceae com 7,99\%, Bignoniaceae com 5,33\%, Ebenaceae com 4,76\%, Salicaceae com 3,88\%, Anacardiaceae com 2,91\% e Urticaceae com 2,42\%. Na Figura 1, encontram-se as famílias com os respectivos números de indivíduos.

No que se refere à riqueza de espécies por família no componente arbóreo, as mais representativas foram: Fabaceae, com 10 espécies, seguida de Myrtaceae e Sapindaceae com seis cada; Anacardiaceae, Saliaceae e Rubiaceae com cinco e Bignoniaceae com 4 espécies.

Quando comparada com outro remanescente de Floresta Estacional Semidecidual, a área não apresentou a mesma riqueza de espécies na mesma ordem listada anteriormente, em que Andrade (2002) relatou que as famílias que se destacaram com maior diversidade de espécies no interior de Pernambuco foram: Fabaceae, seguida de Myrtaceae, Sapindaceae, Euphorbiaceae, Rubiaceae e Lecythidaceae.

Em outra área no interior do Brasil, mais precisamente no interior de Minas Gerais, Marangon (1999) relatou que as famílias que se destacaram com maior diversidade de espécies foram: Fabaceae, seguida de Caesalpiniaceae, Myrtaceae, Euphorbiaceae, Lauraceae, Meliaceae, Mimosaceae, Rubiaceae e Flacourtiaceae.

A análise fitossociológica realizada nos 10.000 $\mathrm{m}^{2}$ de área amostral está representada na Tabela 1 , na qual se visualizam as espécies ordenadas por ordem decrescente de valor de importância (VI), com os respectivos parâmetros calculados.

A respeito da frequência relativa, notou-se que há inversão nos padrões de distribuição das espécies, em que Zanthoxylum rhoifolium se demonstrou mais bem distribuída na área, com 11,53\%, seguida da Campomanesia xanthocarpa, com 11,19\%; da Allophylus edulis, com 7,57\%; da Diospyros brasiliensis, que, apesar de não estar entre as cinco com os maiores números de indivíduos amostrados, se encontrava distribuída em 6,02\% da área amostral; e da Erythroxylum squamatum, com 5,51\%. Juntas, essas espécies apresentaram dominância absoluta de $5,749 \mathrm{~m}^{2} /$ ha, equivalentes a $37,9 \%$, da área total amostrada.

R. Árvore, Viçosa-MG, v.34, n.1, p.103-114, 2010 
Tabela 1 - Parâmetros fitossociológicos calculados para os indivíduos arbóreos (CAP e" $15 \mathrm{~cm})$, em um fragmento de Floresta Estacional Semidecidual em Nazaré da Mata, Pernambuco. Em que: F - Família, DA - densidade absoluta (ind./ha); DR - densidade relativa (\%); FA - frequência absoluta; FR - frequência relativa; DoA - dominância absoluta ( $\left.\mathrm{m}^{2} / \mathrm{ha}\right)$; DoR - dominância relativa; VC - valor de cobertura; e VI - valor de importância.

Table 1 - Phytosociological parameters calculated for tree individuals $\left(C A P e^{\prime 1} 15 \mathrm{~cm}\right)$ in a Fragment of Seasonal Forest in Nazaré da Mata, Pernambuco. Where: F-Family, DA-absolute density (ind. /ha); DR - relative density (\%); FA - absolute frequency; FR - relative frequency; DoA-absolute dominance ( $\left.\mathrm{m}^{2} / \mathrm{ha}\right)$; DoR - relative dominance; $V C$ - cover value and VI-value of importance.

\begin{tabular}{|c|c|c|c|c|c|c|c|c|}
\hline Espécie & Família & DA & DR & FA & FR & DoA & DoR & VI \\
\hline Campomanesia xanthocarpa O. Berg & Myrtaceae & 231 & 18,66 & 65 & 11,19 & 2,053 & 13,56 & 43,41 \\
\hline Zanthoxylum rhoifolium Lam. & Rutaceae & 165 & 13,33 & 67 & 11,53 & 1,778 & 11,75 & 36,61 \\
\hline Anadenanthera colubrina (Vell.) Brenan & Fabaceae & 52 & 4,2 & 20 & 3,44 & 1,594 & 10,53 & 18,17 \\
\hline $\begin{array}{l}\text { Allophylus edulis (A. St. -Hil., A.Juss. } \\
\text { \& Cambess.) Radlk. }\end{array}$ & Sapindaceae & 73 & 5,9 & 44 & 7,57 & 0,580 & 3,83 & 17,30 \\
\hline Erythroxylum squamatum $\mathrm{Sw}$. & Erythroxylaceae & 74 & 5,98 & 32 & 5,51 & 0,817 & 5,4 & 16,88 \\
\hline Diospyros brasiliensis Mart. & Ebenaceae & 59 & 4,77 & 35 & 6,02 & 0,521 & 3,44 & 14,23 \\
\hline $\begin{array}{l}\text { Albizia polycephala (Benth.) Killip } \\
\text { ex Record }\end{array}$ & Fabaceae & 38 & 3,07 & 16 & 2,75 & 0,946 & 6,25 & 12,07 \\
\hline Zizyphus joazeiro Mart. & Rhamnaceae & 30 & 2,42 & 18 & 3,1 & 0,503 & 3,33 & 8,84 \\
\hline Myracrodruon urundeuva Allemao & Anacardiaceae & 26 & 2,1 & 20 & 3,44 & 0,442 & 2,92 & 8,46 \\
\hline Myrciaria tenella (DC.) O. Berg & Myrtaceae & 39 & 3,15 & 16 & 2,75 & 0,169 & 1,12 & 7,02 \\
\hline Machaerium aculeatum Raddi & Fabaceae & 23 & 1,86 & 17 & 2,93 & 0,321 & 2,12 & 6,90 \\
\hline $\begin{array}{l}\text { Tabebuia impetiginosa (Mart. } \\
\text { Ex DC.) Standl. }\end{array}$ & Bignoniaceae & 24 & 1,94 & 16 & 2,75 & 0,316 & 2,09 & 6,77 \\
\hline $\begin{array}{l}\text { Tabebuia avellanadae Lorentz } \\
\text { ex Griseb. }\end{array}$ & Bignoniaceae & 25 & 2,02 & 15 & 2,58 & 0,326 & 2,16 & 6,75 \\
\hline Cecropia palmata Willd. & Urticaceae & 30 & 2,42 & 12 & 2,07 & 0,264 & 1,74 & 6,23 \\
\hline Fabaceae 1 & Fabaceae & 15 & 1,21 & 10 & 1,72 & 0,459 & 3,03 & 5,96 \\
\hline $\begin{array}{l}\text { Samanea tubulosa (Benth.) Barneby } \\
\& \text { J. W. Grimes }\end{array}$ & Fabaceae & 24 & 1,94 & 8 & 1,37 & 0,279 & 1,84 & 5,14 \\
\hline Casearia sylvestris $\mathrm{Sw}$ & Salicaceae & 24 & 1,94 & 6 & 1,03 & 0,24 & 1,58 & 4,55 \\
\hline $\begin{array}{l}\text { Parkia pendula (Willd.) Benth. } \\
\text { Ex Walp. }\end{array}$ & Fabaceae & 8 & 0,65 & 5 & 0,86 & 0,415 & 2,74 & 4,24 \\
\hline Erythroxylum pelleterianum A.St.-Hill. & Erythroxylaceae & 25 & 2,02 & 8 & 1,38 & 0,102 & 0,67 & 4,06 \\
\hline Casearia sp. & Salicaceae & 14 & 1,13 & 11 & 1,89 & 0,132 & 0,87 & 3,89 \\
\hline Indeterminada 3 & - & 15 & 1,21 & 9 & 1,55 & 0,167 & 1,11 & 3,86 \\
\hline Cupania vernalis Cambess. & Sapindaceae & 16 & 1,29 & 11 & 1,89 & 0,094 & 0,62 & 3,80 \\
\hline Lamiaceae 1 & Lamiaceae & 14 & 1,13 & 6 & 1,03 & 0,198 & 1,31 & 3,47 \\
\hline Protium heptaphyllum (Aubl.) & Burseraceae & 11 & 0,89 & 4 & 0,69 & 0,283 & 1,87 & 3,44 \\
\hline Tabebuia roseo-alba (Ridl.) Sandwith & Bignoniaceae & 12 & 0,97 & 7 & 1,2 & 0,164 & 1,09 & 3,26 \\
\hline Guazuma ulmifolia Lam. & Malvaceae & 16 & 1,29 & 6 & 1,03 & 0,102 & 0,67 & 2,99 \\
\hline Myrtaceae 2 & Myrtaceae & 10 & 0,81 & 6 & 1,03 & 0,09 & 0,59 & 2,43 \\
\hline $\begin{array}{l}\text { Tabebuia serratifolia (Vahl) G. } \\
\text { Nicholson }\end{array}$ & Bignoniaceae & 5 & 0,4 & 4 & 0,69 & 0,194 & 1,28 & 2,37 \\
\hline Myrtaceae 1 & Myrtaceae & 9 & 0,73 & 4 & 0,69 & 0,141 & 0,93 & 2,34 \\
\hline Talisia macrophylla Radlk. & Sapindaceae & 8 & 0,65 & 4 & 0,69 & 0,12 & 0,79 & 2,12 \\
\hline Indeterminada 4 & - & 12 & 0,97 & 3 & 0,52 & 0,083 & 0,55 & 2,03 \\
\hline Apeiba tibourbou Aubl. & Malvaceae & 5 & 0,4 & 4 & 0,69 & 0,046 & 0,3 & 1,39 \\
\hline Luehea sp. & Malvaceae & 2 & 0,16 & 2 & 0,34 & 0,129 & 0,85 & 1,35 \\
\hline Alseis floribunda Schott & Rubiaceae & 5 & 0,4 & 3 & 0,52 & 0,062 & 0,41 & 1,32 \\
\hline Annona glabra $L$ & Annonaceae & 2 & 0,16 & 2 & 0,34 & 0,112 & 0,74 & 1,24 \\
\hline Plathymenia foliolosa Benth. & Fabaceae & 7 & 0,57 & 3 & 0,52 & 0,028 & 0,19 & 1,26 \\
\hline Maytenus sp. & Celastraceae & 5 & 0,4 & 4 & 0,69 & 0,017 & 0,11 & 1,20 \\
\hline Indeterminada 2 & - & 6 & 0,48 & 2 & 0,34 & 0,053 & 0,35 & 1,17 \\
\hline
\end{tabular}

R. Árvore, Viçosa-MG, v.34, n.1, p.103-114, 2010 
Tabela 1 - Cont.

Table 1 - Cont.

\begin{tabular}{|c|c|c|c|c|c|c|c|c|}
\hline Xylosma prockia (Turcz.) Turcz. & Salicaceae & 5 & 0,4 & 3 & 0,52 & 0,032 & 0,21 & 1,13 \\
\hline Luehea paniculata Mart. & Malvaceae & 4 & 0,32 & 4 & 0,69 & 0,015 & 0,1 & 1,10 \\
\hline $\begin{array}{l}\text { Myrciaria sp. } \\
\text { Spondias mombin L. }\end{array}$ & $\begin{array}{c}\text { Myrtaceae } \\
\text { Anacardiaceae }\end{array}$ & $\begin{array}{l}5 \\
3\end{array}$ & $\begin{array}{c}0,4 \\
0,24\end{array}$ & $\begin{array}{l}3 \\
2\end{array}$ & $\begin{array}{l}0,52 \\
0,34\end{array}$ & $\begin{array}{c}0,027 \\
0,07\end{array}$ & $\begin{array}{l}0,18 \\
0,46\end{array}$ & $\begin{array}{l}1,10 \\
1,05\end{array}$ \\
\hline Tapirira guianensis Aubl. & Anacardiaceae & 3 & 0,24 & 2 & 0,34 & 0,064 & 0,42 & 1,00 \\
\hline Indeterminada 1 & - & 5 & 0,4 & 2 & 0,34 & 0,036 & 0,24 & 0,98 \\
\hline Guapira opposita (Vell.) Reitz. & Nyctaginaceae & 4 & 0,32 & 2 & 0,34 & 0,038 & 0,25 & 0,92 \\
\hline Sapindus saponaria $L$. & Sapindaceae & 5 & 0,4 & 2 & 0,34 & 0,026 & 0,17 & 0,92 \\
\hline Cassia grandis L. $f$. & Fabaceae & 1 & 0,08 & 1 & 0,17 & 0,077 & 0,51 & 0,76 \\
\hline Talisia esculenta (A. St.-Hil.) Radlk. & Sapindaceae & 3 & 0,24 & 2 & 0,34 & 0,025 & 0,16 & 0,74 \\
\hline Inga laurina (Sw.) Willd. & Fabaceae & 2 & 0,16 & 2 & 0,34 & 0,034 & 0,23 & 0,73 \\
\hline Ochnaceae 1 & Ochnaceae & 3 & 0,24 & 2 & 0,34 & 0,018 & 0,12 & 0,70 \\
\hline Licania sp. & Chrysobalanaceae & 3 & 0,24 & 2 & 0,34 & 0,014 & 0,09 & 0,68 \\
\hline Mangifera indica $L$. & Anacardiaceae & 1 & 0,08 & 1 & 0,17 & 0,067 & 0,44 & 0,69 \\
\hline Myrcia sylvatica (G.Mey.) DC. & Myrtaceae & 2 & 0,16 & 2 & 0,34 & 0,03 & 0,2 & 0,70 \\
\hline Brunfelsia uniflora (Pohl) D. Don & Solanaceae & 3 & 0,24 & 2 & 0,34 & 0,008 & 0,05 & 0,64 \\
\hline Casearia arborea (Rich.) Urb. & Salicaceae & 3 & 0,24 & 2 & 0,34 & 0,006 & 0,04 & 0,62 \\
\hline Siparuna sp. & Siparunaceae & 2 & 0,16 & 2 & 0,34 & 0,014 & 0,09 & 0,59 \\
\hline Anonnaceae 1 & Annonaceae & 1 & 0,08 & 1 & 0,17 & 0,044 & 0,29 & 0,54 \\
\hline Astronium fraxinifolium Schott \& Spreng. & Anacardiaceae & 3 & 0,24 & 1 & 0,17 & 0,017 & 0,11 & 0,52 \\
\hline Cupania racemosa (Vell.) Radlk. & Sapindaceae & 3 & 0,24 & 1 & 0,17 & 0,021 & 0,14 & 0,55 \\
\hline Salicaceae 1 & Salicaceae & 2 & 0,16 & 2 & 0,34 & 0,005 & 0,03 & 0,54 \\
\hline Rubiaceae 1 & Rubiaceae & 2 & 0,16 & 2 & 0,34 & 0,004 & 0,03 & 0,53 \\
\hline Guettarda platypoda $D C$. & Rubiaceae & 1 & 0,08 & 1 & 0,17 & 0,03 & 0,2 & 0,45 \\
\hline Hymenaea courbaril L. & Fabaceae & 1 & 0,08 & 1 & 0,17 & 0,019 & 0,12 & 0,37 \\
\hline Jathropha mollissima (Pohl) Baill. & Euphorbiaceae & 1 & 0,08 & 1 & 0,17 & 0,013 & 0,09 & 0,33 \\
\hline Vitex rufescens A. Juss. & Lamiaceae & 1 & 0,08 & 1 & 0,17 & 0,011 & 0,07 & 0,32 \\
\hline Genipa americana $L$. & Rubiaceae & 1 & 0,08 & 1 & 0,17 & 0,007 & 0,05 & 0,3 \\
\hline $\begin{array}{l}\text { Ocimum gratissimum L. } \\
\text { Euphorbiaceae } 1\end{array}$ & $\begin{array}{c}\text { Lamiaceae } \\
\text { Euphorbiaceae }\end{array}$ & $\begin{array}{l}1 \\
1\end{array}$ & $\begin{array}{l}0,08 \\
0,08\end{array}$ & $\begin{array}{l}1 \\
1\end{array}$ & $\begin{array}{l}0,17 \\
0,17\end{array}$ & $\begin{array}{l}0,007 \\
0,004\end{array}$ & $\begin{array}{l}0,05 \\
0,03\end{array}$ & $\begin{array}{l}0,29 \\
0,27\end{array}$ \\
\hline Guapira sp. & Nyctaginaceae & 1 & 0,08 & 1 & 0,17 & 0,005 & 0,03 & 0,28 \\
\hline Manilkara salzmannii (A.DC.) H.J.Lam & Sapotaceae & 1 & 0,08 & 1 & 0,17 & 0,004 & 0,03 & 0,27 \\
\hline Randia nitida $D C$ & Rubiaceae & 1 & 0,08 & 1 & 0,17 & 0,002 & 0,01 & 0,26 \\
\hline Triplaris surinamensis Cham. & Polygonaceae & 1 & 0,08 & 1 & 0,17 & 0,004 & 0,02 & 0,27 \\
\hline Total & & 1238 & 100 & 581 & 100 & 15,135 & 100 & 300 \\
\hline
\end{tabular}

Para a área basal, calculou-se um valor referente a $15,135 \mathrm{~m}^{2} / \mathrm{ha}$, estando um pouco inferior ao calculado por Lopes (2007), em estudo da caracterização fisionômico-estrutural em fragmento de mata seca em Pernambuco, onde ele encontrou uma área basal de $18,7 \mathrm{~m}^{2} / \mathrm{ha}$. Ao confrontar os valores no interior do Pernambuco com o calculado por Feliciano (1999) em estudo realizado na Estação Ecológica de São Carlos, em uma área de Floresta Estacional Semidecidual (45,98 $\mathrm{m}^{2} / \mathrm{ha}$ ), percebeu-se que havia discrepância nos valores. Vale ressaltar que tal fato pode ser explicado pelo clima distinto, como também pelas classes de solos diferentes de cada fragmento.

Em relação às 10 espécies com maiores valores de importância, foi observado que a ordem não apareceu na mesma sequência do valor de cobertura, e que, juntas, as espécies Campomanesia xanthocarpa, Zanthoxylum rhoifolium, Anadenanthera colubrina, Allophylus edulis, Erythroxylum squamatum, Diospyros brasiliensis, Albizia polycephala, Zizyphus joazeiro, Myracrodruon urundeuva e Myrciaria tenella se equivaliam a $61 \%$ dos indivíduos amostrados na área (Figura 2).

O que melhor explica a maior predominância e distribuição das espécies observadas na Figura 1 devem ser as características ecológicas de cada espécie, em que a grande parte era de decíduas, ou seja, espécies que perdem suas folhas em períodos secos como forma de adaptação para minimizar a perda de água pela evapotranspiração.

R. Árvore, Viçosa-MG, v.34, n.1, p.103-114, 2010 
O fato de essas espécies apresentarem maiores densidades pode estar relacionado com as características favoráveis do ambiente, propiciando ao mesmo tempo maior estabelecimento destas, pois, teoricamente, a espécie mais importante é aquela que consegue maior sucesso ao explorar os recursos do hábitat.

A Mata do Alcaparra, por estar próxima a uma cidade e algumas comunidades e não ser dada atenção especial à sua conservação, vem sofrendo pressões antrópicas constantemente. Apesar de todos esses fatores influenciando a dinâmica da área, percebeu-se que havia riqueza de espécies arbóreas (72), o que pode ser um valor consideravelmente bom quando comparado com o do trabalho de Andrade (2002), com 88 espécies arbóreas; e de Lopes (2007), com 79 espécies arbóreas levantadas em fragmento de Floresta Estacional Semidecidual, no interior de Pernambuco. Valores esses um pouco diferente dos encontrados por Longhi et al. (2000), com 64 espécies arbóreas e arbustivas levantadas; Werneck et al. (2000), 68 ; e um pouco inferior quando comparados com os do trabalho de Silva et al. (2000), com 91, sendo todos esses estudos realizados em fragmentos de Floresta Estacional Semidecidual, porém em diferentes áreas fitogeográficas.

É importante, contudo, ressaltar que os diferentes níveis de inclusão estabelecidos, com as diferenças nos esforços amostrais estudados, são fatores que devem ser analisados criteriosamente, para não se fazerem inferências imprecisas de determinadas áreas. Além disso, ressalvando-se tais diferenças metodológicas, estudos realizados em florestas tropicais indicam que o estádio sucessional e o histórico de perturbação da floresta podem influenciar o número de espécies encontradas na comunidade (TABARELLI e MANTOVANI, 1999; WERNECK et al., 2000).

Em relação à distribuição diamétrica, o remanescente florestal apresentou distribuição no formato de Jinvertido, comum em florestas inequiâneas, concentrando maior número de indivíduos nas primeiras classes de diâmetro, conforme se pode observar na Figura 3.

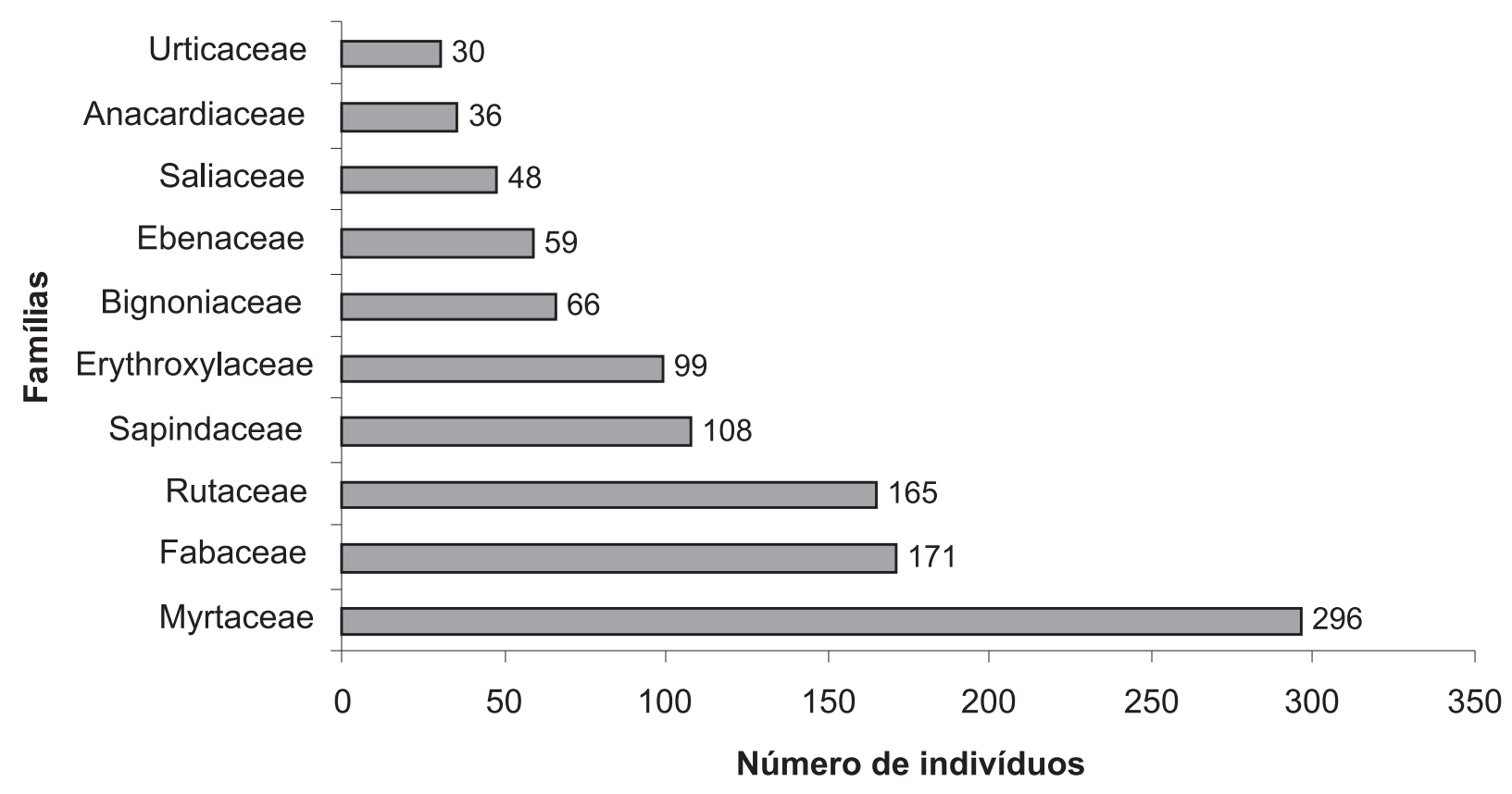

Figura 1 - Famílias com maior representatividade em número de indivíduos arbóreos, em um fragmento de Floresta Estacional Semidecidual de Nazaré da Mata, Pernambuco.

Figure 1 - Families with greater representativeness in number of trees in a fragment of seasonal forest, Nazaré da Mata, Pernambuco.

R. Árvore, Viçosa-MG, v.34, n.1, p.103-114, 2010 


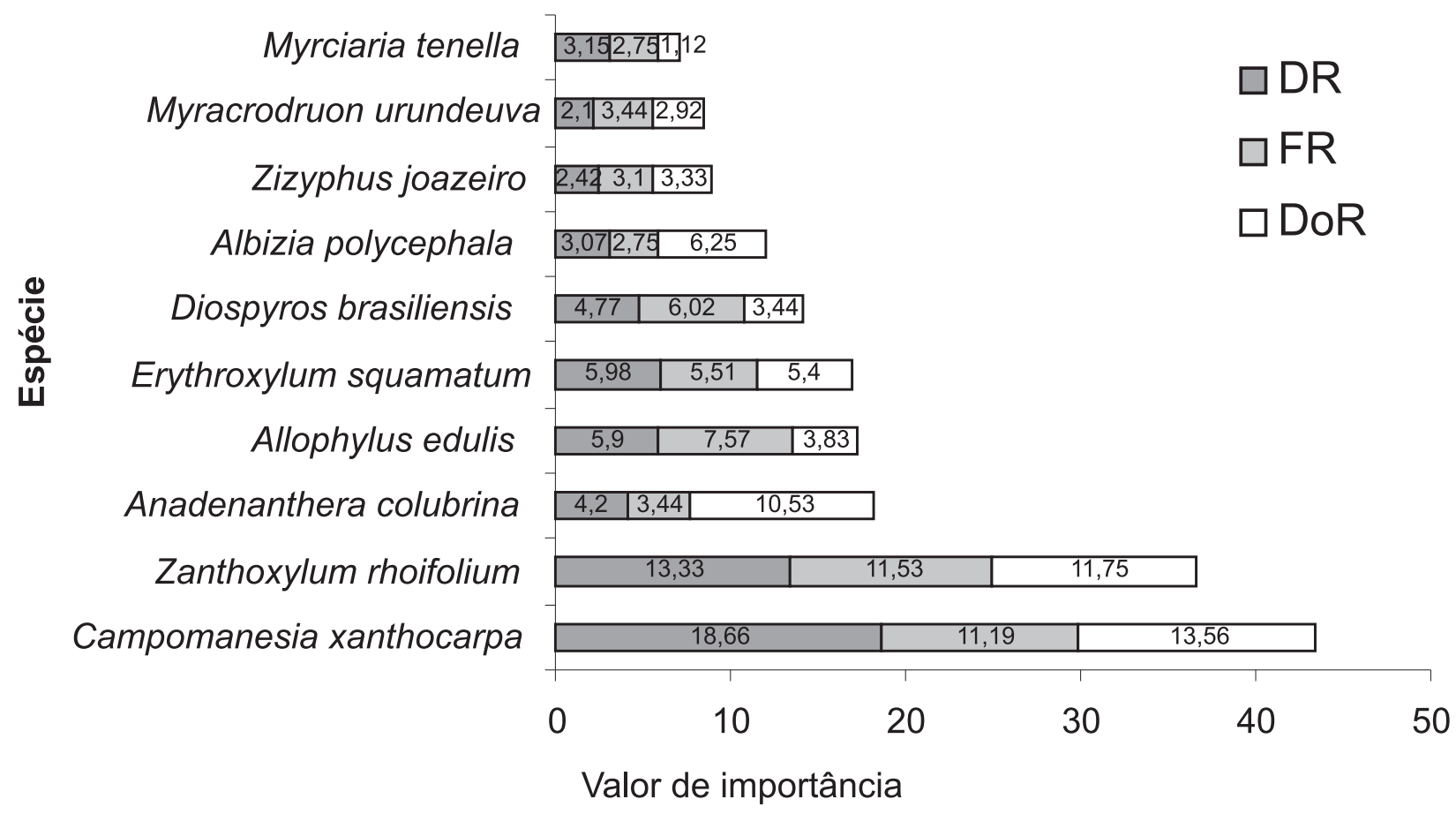

Figura 2 - Dez espécies com os maiores valores de importância, representados pelas somas dos parâmetros densidade relativa, frequência relativa e dominância relativa amostrados em fragmento de Floresta Estacional Semidecidual, em Nazaré da Mata, Pernambuco.

Figure 2 - Ten species with the largest values of importance, represented by the sum of parameters, relative density, relative frequency and relative dominance sampled in a Fragment of Seasonal Forest, Nazaré da Mata, Pernambuco.

Ao analisar a Figura 3, nota-se que 56,9\% dos indivíduos amostrados foram encontrados nos dois primeiros centros de classe. Com base nesses dados, é possível afirmar que o fragmento Mata do Alcaparra é uma área de floresta secundária, pela grande quantidade de indivíduos amostrados nos primeiros centros de classe e também pelo fato de ser comum encontrar em seu interior muitas árvores recém-cortadas, tornando evidente a presença de tocos em seu interior. A partir desses dados, pode-se inferir que a área estava em estágio de desenvolvimento, tomando como base o grande número de indivíduos encontrados com o diâmetro mínimo considerado $(4,77 \mathrm{~cm})$.

Alguns pesquisadores, como Scolforo et al. (1998), relataram que a análise dos dados de distribuição de diâmetros é importante, pois pode predizer sobre o passado (perturbações, como exploração da madeira) e o futuro da floresta (como estoque de madeira disponível e informações sobre uma possível reposição florestal).
Para o estrato arbóreo do fragmento em estudo, constatou-se que as alturas variaram entre 2,5 e 18 m e que o dossel possuía indivíduos arbóreos com alturas superiores a 13,5 m, a exemplo da Casearia sylvestris e Albizia polycephala com 14 m, Tabebuia roseo-alba e Tabebuia serratifolia com $16 \mathrm{~m}$ e Anadenanthera colubrina, cuja altura máxima alcança $18 \mathrm{~m}$. Na distribuição dos indivíduos por classe de altura, a maior parte foi encontrada no segundo centro de classe (Figura 4), o que correspondeu a 77,5\% dos indivíduos amostrados.

Analisando os valores de área basal total entre as classes de altura, visualizou-se que a primeira classe de altura correspondente a 4,32 d" $\mathrm{H}<9,16 \mathrm{~m}$ apresentou o maior valor $\left(8,846 \mathrm{~m}^{2} / \mathrm{ha}\right)$; e, ao observar à terceira classe de altura equivalente às árvores com $\mathrm{H}$ e" 9,16 $\mathrm{m}$, percebeu-se pequena redução na área basal $(5,816$ $\left.\mathrm{m}^{2} / \mathrm{ha}\right)$.

R. Árvore, Viçosa-MG, v.34, n.1, p.103-114, 2010 


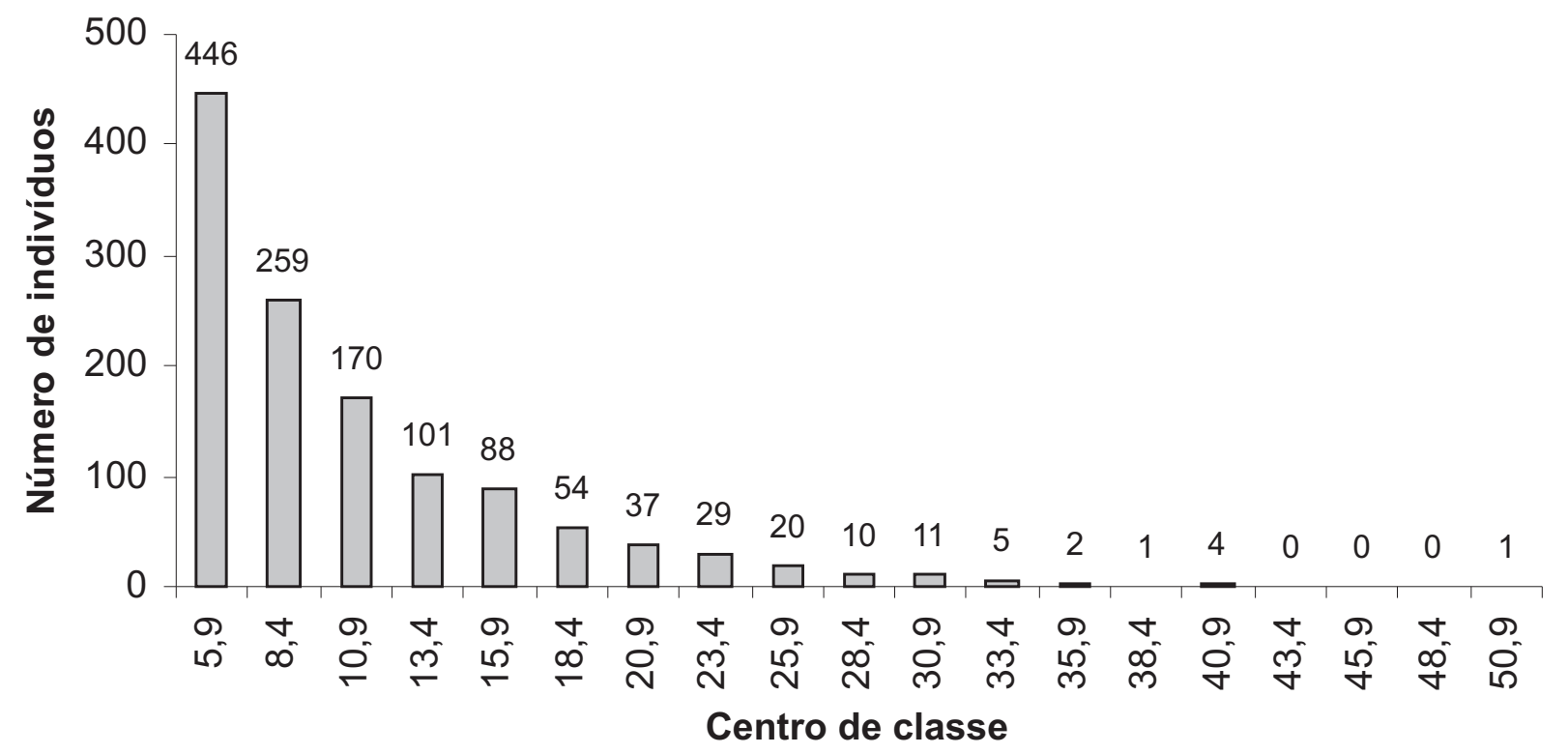

Figura 3 - Distribuição diamétrica dos indivíduos arbóreos amostrados em um fragmento de Floresta Estacional Semidecidual em Nazaré da Mata, Pernambuco.

Figure 3 - Diametric distribution in a Fragment of Seasonal Forest, Nazaré da Mata, Pernambuco.

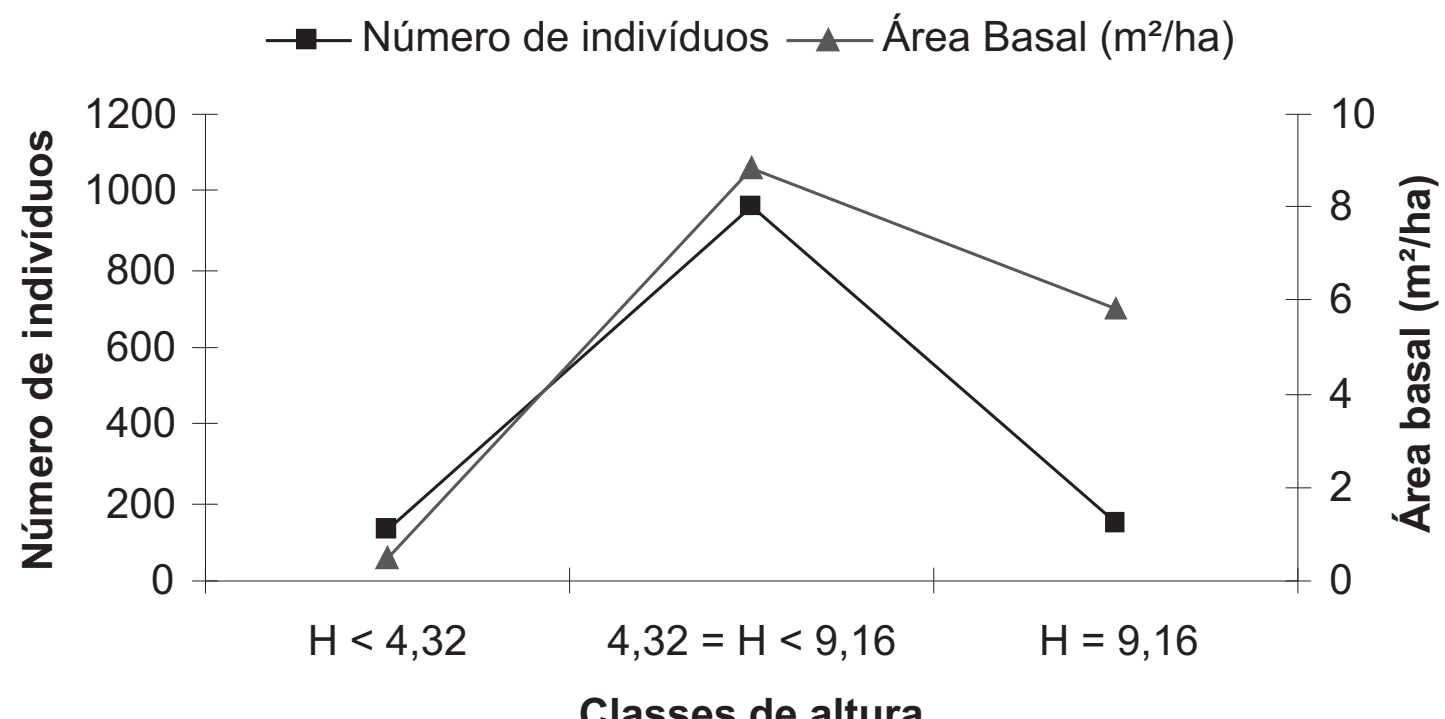

Figura 4 - Distribuição dos indivíduos e área basal em classes de altura em um fragmento de Floresta Estacional Semidecidual em Nazaré da Mata, Pernambuco.

Figure 4-Distribution of individuals and basal area in height classes in a Fragment of Seasonal Forest, Nazaré da Mata,

R. Árvore, Viçosa-MG, v.34, n.1, p.103-114, 2010 


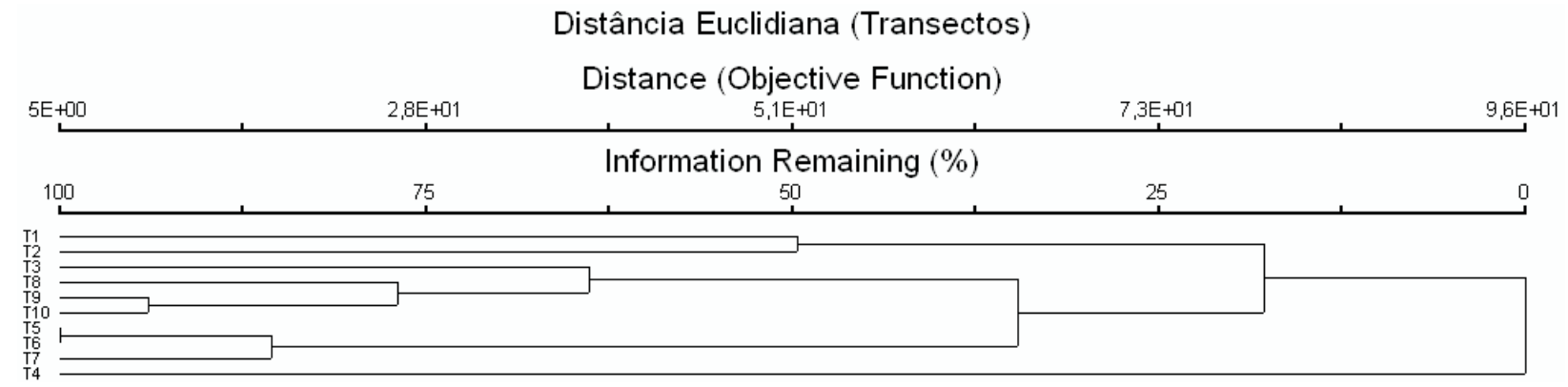

Figura 5-Dendograma de dissimilaridade pelo Método de Ward, baseado na distância euclidiana entre os 10 transectos arbóreos amostrados, no fragmento de Floresta Estacional Semidecidual, em Nazaré da Mata, Pernambuco.

Figure 5 - Dissimilarity Dendrogram by the Ward Method based on the Euclidean distance between ten tree sampled transects, in a fragment of Seasonal Forest, Nazaré da Mata, Pernambuco.

DCA

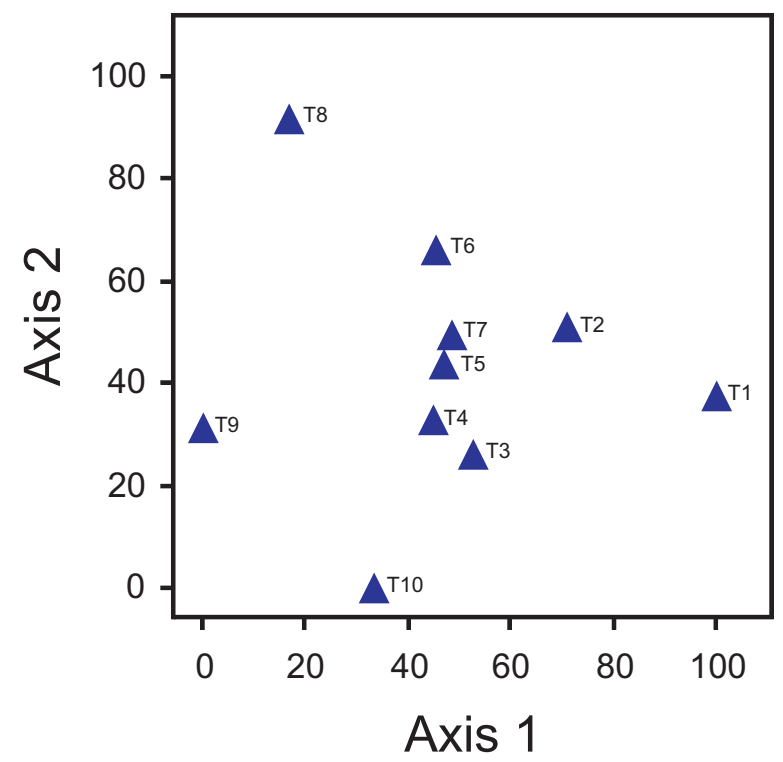

Figura 6 - Diagrama de ordenação nos dois primeiros eixos da análise de correspondência retificada (DCA) dos dados florísticos do fragmento florestal Mata do Alcaparra, em Nazaré da Mata, Pernambuco.

Figure 6-Ordination diagram in the first two axes of the analysis of rectified correspondence (DCA) for floristic data from the forest fragment "Mata do Alcaparra”, Nazaré da Mata, Pernambuco.

Ao avaliar a estrutura vertical juntamente com a estrutura diamétrica, pôde-se considerar que o fragmento se encontrava, provavelmente, em estágio inicial de sucessão, porque concentrou maior número de indivíduos nas primeiras classes. É válido ressaltar que a sucessão é um processo ecológico que evolui lentamente e, em muitos casos, depende do estado de conservação da área.

Diante disso, Sanqueta (1995) relatou que o estudo da estratificação da floresta como um todo possibilita obter informações para a compreensão das características da estrutura vertical do fragmento, o que pode dar embasamento para o entendimento das estratégias de regeneração natural, crescimento e sobrevivência da população.

O índice de diversidade de Shannon-Weaner (H'), calculado para o fragmento Mata do Alcaparra, foi de 3,29 nats/ind, valor um pouco inferior ao encontrado por Andrade (2002), 3,42 nats/ind.; e Feliciano (1999), 3,55 nats/ind, também estudando uma área de Floresta Estacional Semidecidual.

A respeito da similaridade florística, visualiza-se, na Figura 5, a formação de quatro grupos, tomando como base a linha de Fenon, que, segundo Souza et al. (1997), éo tracejo de uma linha perpendicular ao eixo do dendograma no nível de 50\%, no qual intercepta o número de ramos, sendo o número de ramos interceptados o número de grupamentos formados.

Uma das razões que podem explicar a dissimilaridade em torno de $50 \%$ dos transectos 1 e 2, já que estão próximos, diz respeito ao estado de conservação em que ambos se encontravam, em que o transecto 2 apresentava o interior um pouco perturbado, pois se verificaram algumas clareiras e uma trilha, comprometendo, assim, parte de sua vegetação. Já no transecto 1 não foi constatado nenhum tipo de perturbação, pois se localizava na face sul do fragmento com número elevado de indivíduos adensados, o que, em princípio, dificultaria o acesso ao local.

R. Árvore, Viçosa-MG, v.34, n.1, p.103-114, 2010 
Uma das razões que explicam a dissimilaridade entre os grupos T3, T8, T9 e T10 (segundo grupo), quando comparados com T5, T6 e T7 (terceiro grupo), deve-se ao formato observado no fragmento, pois o segundo grupo se encontrava na face sul, com formato desuniforme tendendo a retangular; e, quando foi feita a medição de distâncias entre o lado leste e o oeste, observou-se uma extensão em torno de 200 m. Já o terceiro grupo se encontrava na face norte da área, em que foi verificado um formato arredondado (círculo), e, quando foram medidas as distâncias entre os lados leste e oeste, constatou-se algo em torno de $650 \mathrm{~m}$, o que teoricamente pode ter influenciado na ocorrência de diferentes espécies nessas áreas.

Um dos fatos que melhor explicam a formação dos grupos e a heterogeneidade entre os transectos diz respeito à ocorrência exclusiva de determinadas espécies em apenas um transecto, que é o caso do T-4, onde foram encontradas Astronium flaxinifolium, Annona glabra, Protium heptaphyllum, Erythroxylum pelleterianum, Indeterminada 2, Ocimum gratissimum, Myrtaceae 1, Manilkara salzmannii, Brunfelsia uniflora, Luehea sp. e Vitex rufescens.

Existem diversos fatores que podem influenciar a ocorrência dessas espécies apenas nesse local, os quais podem ser desde os atributos químicos e físicos do solo, como também os agentes dispersores e polinizadores, entre outros. O fato é que no interior do transecto 4 existe um córrego, de aproximadamente 2,5 $\mathrm{m}$ de largura, que proporciona maior umidade ao local, o que é muito provável que possa vir a influenciar a ocorrência dessas espécies.

Na Figura 6, apresenta-se o diagrama de ordenação da análise de correspondência, em que se verificam as diferenças florísticas entre os transectos. Na análise, encontraram-se autovalores baixos nos dois primeiros eixos de ordenação $(0,319$ e 0,177$)$, ou seja, as espécies, em sua maioria, estavam distribuídas por todo o gradiente, e uma minoria concentrava-se em setores particulares do gradiente (TER BRAAK, 1995).

O que melhor explica essas diferenciações florísticas entre cada transecto é o fato de alguns estarem em melhor estado de conservação que os outros e, consequentemente, apresentarem estágios sucessionais diferenciados, pois a Mata do Alcaparra apresentava perturbada, sendo encontrada em seu interior diversas trilhas e formações de clareiras devido à retirada de lenha ilegal. Para ter certeza de que a diversidade da área se mantenha e possa expandir, é necessário intensificar a vigilância, no intuito de coibir a retirada de lenha e punir os culpados, como medida de repressão para garantir toda a diversidade biológica da área.

\section{CONCLUSÃO}

As espécies Campomanesia xanthocarpa, Zanthoxylum rhoifolium e Anadenanthera colubrina foram as que apresentaram os maiores valores de importância, demonstrando, dessa forma, melhor adaptação às condições locais.

O fragmento florestal encontrava-se com grande diversidade de espécies vegetais na comunidade arbórea, apesar de todos os tipos de intervenções que vinha sofrendo.

A alta dissimilaridade entre os transectos é reflexo das formações de micro-hábitats em seu interior devido às perturbações encontradas na área.

\section{REFERÊNCIAS}

AGENDA 21 DE NAZARÉ DA MATA. No

caminho do desenvolvimento sustentável. Nazaré da Mata: 2004. 75p.

AGUILAR, R.; GALLETO, L. Effects of forest fragmentation on male and female reproductive success in Cestrum parqui (Solanaceae). Oecologia, v.138, n.4, p.513-520, 2004.

ANDRADE, K. V. S. A. Fisionomia e estrutura de um remanescente de floresta estacional semidecidual na estação ecológica do Tapacurá, município de São Lourenço da Mata, Pernambuco - Brasil. 2002. $70 f$.

Dissertação (Mestrado em Botânica) Universidade Federal Rural de Pernambuco, Recife, 2002.

BRASIL. Ministério do Meio Ambiente. Fragmentação de ecossistemas: causas, efeitos sobre a biodiversidade e recomendações de políticas públicas. Brasília: 2003. 508p.

CAUSTON, D. R. An introduction to vegetation analysis, principles and interpretation. London: Unwin Hyman, 1988. 342p. 
EMPRESA BRASILEIRA DE PESQUISA AGROPECUARIA - EMBRAPA. Centro Nacional de Pesquisa de Solos. Sistema brasileiro de classificação de solos. Rio de Janeiro: 1999. 412p.

FELICIANO, A. L. P. Caracterização ambiental, florística e fitossociológica de uma unidade de conservação. Caso de estudo: Estação ecológica de São Carlos, Brotas, SP. 1999. 157f. Tese (Doutorado em

Ecologia e Recursos Naturais) - Universidade Federal de São Carlos, São Carlos, 1999.

LONGHI, S. J. et al. Aspectos fitossociológicos de fragmento de floresta estacional decidual, Santa Maria, RS. Ciência Florestal, v.10, n.2, p.59$74,2000$.

LOPES, C. G. R. Relações florísticas e estruturais entre fragmentos de florestas secas e úmidas (Floresta Atlântica), Nordeste do Brasil. 2007. $85 \mathrm{f}$. Dissertação (Mestrado em Botânica) -

Universidade Federal Rural de Pernambuco, Recife, 2007.

MARANGON, L. C. Florística e fitossociologia de área de floresta estacional semidecidual visando dinâmica de espécies florestais arbóreas no município de Viçosa, MG. 1999. 139f. Tese (Doutorado em Ecologia e Recursos Naturais) - Universidade Federal de São Carlos, São Carlos, 1999.

MARTINS, F. R. Estrutura de uma floresta mesófila. Campinas: Universidade de Campinas, 1993. 245p.

MCCUNE, B.; MEFFORD, M. J. PC-ORD version 4.14: Multivariate analysis of ecological data. Glaneden Beach: MjM Software Design, 1999. 237p.

METZGER, J. P. Tree functional group richness and landscape structure in Brazilian tropical fragmented landscape. Ecological Applications, v.10, n.4, p.1147-1161, 2000.

MUELLER-DOMBOIS, D.; ELLEMBERG, H. Aims and methods of vegetation ecology. New York: John Wiley \& Sons, 1974. 547p.
RESTREPO, C.; GOMEZ, N.; HEREDIAS, S. Anthropogenic edges, treefall gaps, and fruitfrugivore interactions ina neotropical montane forest. Ecology, v.80, n.2, p.668-685, 1999.

RODRIGUES, P. J. F. P.; NASCIMENTO, M. T. Fragmentação florestal: breves considerações teóricos sobre efeito de borda. Rodriguésia, v.57, n.1, p.63-74, 2006.

SANQUETA, C. R. Análise da estrutura vertical de florestas através do diagrama h-M. Ciência Florestal, v.5, n.1, p.55-68, 1995.

SCOLFORO, J. R. S.; PULTZ, F. A.; MELO, J. M. Modelagem da produção, idade das florestas nativa, distribuição espacial das espécies e a análise estrutural. In: SCOLFORO, J. R. S. (Coord.). Manejo florestal. Lavras:UFLA/FAEPE, 1998. p.189-246.

SILVA, A. F.; FONTES, N. L. R.; LEITÃO-FILHO, H. F. Composição florística e estrutura horizontal do estrato arbóreo de um trecho da Mata da Biologia da Universidade Federal de Viçosa Zona da Mata de Minas Gerais. Revista Árvore, v.24, n. 4, p.397-405, 2000.

SILVA, N. R. S. et al. Composição florística e estrutura de uma floresta estacional semidecidual montana em Viçosa, MG. Revista Árvore, v.28, n.3, p.397-405, 2004.

SOUZA, A. L.; FERREIRA, R. L. C.; XAVIER, A. Análise de agrupamento aplicada à área florestal. Viçosa, MG: SIF, 1997. 109p.

VELOSO, H. P. et al. (Org.). Manual técnico da vegetação brasileira. Rio de Janeiro: IBGE/Departamento de Recursos Naturais e Estudos Ambientais, 1992. 93p.

TABARELLI, M.; MANTOVANI, W. A regeneração de uma floresta tropical montana após corte e queima (São Paulo - Brasil). Revista Brasileira de Biologia, v.59, n.2, p. 239-251, 1999.

TANIZAKI, K.; MOULTON, T. P. A fragmentação da Mata Atlântica no estado do Rio de Janeiro e a perda de biodiversidade. In: BERGAllo, H. G. et al. (Orgs.). A fauna ameaçada de extinção do Estado do Rio de Janeiro. Rio de Janeiro: UERJ, 2000. p.135-166.

R. Árvore, Viçosa-MG, v.34, n.1, p.103-114, 2010 
TER BRAAK, C. J. F. Ordination. In: JONGMAN, R. H. G.; TER BRAAK, C. J. F.; van TONGEREN, $O$. F. R. Data analysis in community and landscape ecology. Cambrigde: Cambridge University Press, 1995. p.91-173.
WERNECK, M. S. et al. Florística e estrutura de três trechos de uma floresta semidecídua na Estação Ecológica do Tripuí, Ouro Preto, MG. Revista Brasileira de Botânica, v.23, n.1, p.97-106, 2000. 